\title{
A preoperative nomogram predicting the pseudocapsule status in localized renal cell carcinoma
}

\author{
Jiao Hu ${ }^{1}$, Jinbo Chen ${ }^{1}$, Huihuang $\mathrm{Li}^{1}$, Tongchen $\mathrm{He}^{1}$, Hao Deng ${ }^{1}$, Guanghui Gong ${ }^{2}$, Yu Cui ${ }^{1}$, Peihua Liu ${ }^{1}$, \\ Wenbiao Ren ${ }^{1}$, Xu Zhou ${ }^{3}$, Chao $\mathrm{Li}^{1}$, Xiongbing $\mathrm{Zu}^{1}$ \\ ${ }^{1}$ Department of Urology, ${ }^{2}$ Department of Pathology, ${ }^{3}$ Reproductive Medicine Center, Xiangya Hospital, Central South University, Changsha 410008 , \\ China \\ Contributions: (I) Conception and design: J Hu, C Li, X Zu; (II) Administrative support: None; (III) Provision of study materials or patients: C Li, X \\ Zu; (IV) Collection and assembly of data: Jiao Hu, T He, H Deng, G Gong, Y Cui, P Liu, C Li; (V) Data analysis and interpretation: J Hu, C Li, W \\ Ren, H Li, X Zhou; (VI) Manuscript writing: All authors; (VII) Final approval of manuscript: All authors. \\ Correspondence to: Xiongbing Zu; Chao Li. Department of Urology, Xiangya Hospital, Central South University, Changsha 410008, China. \\ Email: whzuxb@163.com; li4307chao@126.com.
}

\begin{abstract}
Background: Tumor enucleation (TE) surgery for localized renal cell carcinoma (RCC) relies on a complete peritumoral pseudocapsule (PC). Study objective was to develop a preoperative model to predict PC status.

Methods: The prediction model was developed in a cohort that consisted of 170 patients with localized RCC, and data was gathered from 2010 to 2015. Multivariable logistic regression analysis and R were used to generate this prediction model. The statistical performance was assessed with respect to the calibration, discrimination, and clinical usefulness.

Results: The prediction model incorporated the systemic inflammatory markers [neutrophil-lymphocyte ratio (NLR); albumin-globulin ratio (AGR)], CT imaging features (tumor size and necrosis), and clinical risk factors (BMI). The model showed good discrimination, with a C-index of 0.85 (0.78-0.91), and good calibration $(\mathrm{P}=0.60)$. The sensitivity and specificity were $62 \%$ and $94 \%$ respectively. Decision curves and clinical impact curve demonstrated that the current model was clinically useful.

Conclusions: We constructed a model that incorporated both the systematic inflammatory markers and clinical risk factors. It can be conveniently used to preoperatively predict the individualized risk of PC invasion and identify the best candidates to receive TE surgery.
\end{abstract}

Keywords: Renal cell carcinoma (RCC); pseudocapsule invasion; nomogram

Submitted Sep 22, 2019. Accepted for publication Jan 04, 2020.

doi: $10.21037 /$ tau.2020.01.26

View this article at: http://dx.doi.org/10.21037/tau.2020.01.26

\section{Introduction}

Nephron sparing surgery (NSS) is recommended as the first choice for patients with localized renal cell carcinoma (RCC) (1). Standardized NSS excises renal cancers with a layer of normal renal parenchyma to achieve negative surgical margin. But the excision of normal renal parenchyma may cause damage to renal function (2). To optimize the renal function preservation, tumor enucleation (TE) technique has been developed by excising the tumor without normal parenchyma along a natural cleavage between the peritumoral pseudocapsule (PC) and healthy parenchyma (3-5). Recently, Dell'Atti et al. developed an unclamped sutureless laparoscopic simple enucleation technique for renal tumors with low nephrometry score (6). After analyzing the complication rates, functional and oncological outcomes, we found that this innovative technique was a rational and safe approach. However, other studies reported that TE surgery increased recurrence and death due to higher positive surgical margin rate in comparison with 
standardized NSS, especially for patients without PC $(7,8)$.

Peritumoral PC is a layer of fibrous connective tissue surrounding the cancer. A complete $\mathrm{PC}$ can prevent tumor cells from infiltrating adjacent renal parenchyma and facilitate the smooth implementation of TE surgery (9). In contrast, patients with disrupted PC or infiltrated parenchyma are recommended to receive standardized NSS to avoid positive surgical margin. Notably, the PC status is the most critical indication of TE surgery. Previous researches revealed that $\mathrm{PC}$ status was related to several pathological features, such as tumor grade and histological subtype (10). However, these pathological characters are difficult to identify before surgery. Hence, it is urgent to identify preoperative predictors of $\mathrm{PC}$ status.

It has been well summarized that systemic inflammatory markers, such as neutrophil-lymphocyte ratio (NLR), platelet-lymphocyte ratio (PLR), lymphocyte-monocyte ratio (LMR) and albumin-globulin ratio (AGR), are associated with pathological characters and prognosis of multiple cancers, such as renal cancer, gastric cancer, bladder cancer and prostate cancer (11-15). Importantly, these markers can be simply and economically assessed before surgery. However, there is no study to explore the associations between these inflammatory markers and PC status. Hence, we performed this retrospective study to construct a preoperative prediction model for PC status by incorporating systematic inflammatory and clinical characters.

\section{Methods}

\section{Study population and clinical data}

This study obtained approval from our institutional ethical review board (Ethical approval ID: 201912530). The informed consent was waived for this study. A total of 250 consecutive patients with localized RCC were analyzed from 2010 to 2015. All patients received laparoscopic NSS which excised a layer of normal renal parenchyma with a thickness of $5 \mathrm{~mm}$. Conversion to open surgery was not necessary in any patient. All surgical procedures were completed by skillful surgeons with more than five years of laparoscopic surgical experience. The criteria and flow diagram of patient inclusion were shown in Figure S1. Clinical data were collected from archived records, including hematological parameters, age, gender, body mass index (BMI) and smoking history, and some systemic comorbidities (chronic renal disease, hypertension, and coronary heart disease). Patients would be excluded if they had diseases which affected hematological parameters, such as cold, fever, pneumonia and urinary tract infection. Blood samples were obtained and examined within three days prior to surgery. The normal references of hematological parameters were provided in Table S1. We calculated four systemic inflammatory indexes including NLR, PLR, LMR and AGR. All patients received computer tomography (CT) examination. Several CT imaging features, such as tumor size, necrosis on CT scan, tumor shape and enhancement pattern, were described with reference to a previous report (Figure S2) (16). Of particular note was the necrosis on CT scan that was defined as a low-density region without contrast enhancement during the cortex or parenchyma phases.

\section{Pathological characters}

Two independent pathologists re-evaluated all histological sections. Tumor grade was evaluated based on Fuhrman grade criteria and was divided into high grade (G3-4) and low grade (G1-2). Histological subtype was assessed according to the World Health Organization 2016 classification. Because all eligible patients were diagnosed with localized RCC, the tumor stage was not further evaluated. Four typical PC status were shown in Figure 1. Complete PC: intact PC without disconnection or neoplastic infiltration; PC absence: PC was not visible at any point in all slides, and the cancer cell directly adjoined the renal parenchyma; PC infiltration: cancer cell infiltrated the PC, but not exceeded it; Parenchyma infiltration: cancer cell infiltrated peritumoral renal parenchyma. Here, we defined PC absence, PC infiltration and parenchyma infiltration as "PC invasion".

\section{Statistical analysis}

Pearson's chi-squared test was used to evaluate associations between categorical variables. Independent-samples $\mathrm{T}$ test was applied to analyzed continuous variables. The optimal cut-off values of systemic inflammatory markers were calculated by using receiver operating characteristic (ROC) curves and Youden index. We identified the risk factors of PC invasion using univariate analysis. Factors which were significant or nearly significant in univariable analysis were reconsidered for further forward stepwise multivariate logistic regression analysis. Then we constructed a graphic nomogram by using R studio (rms 

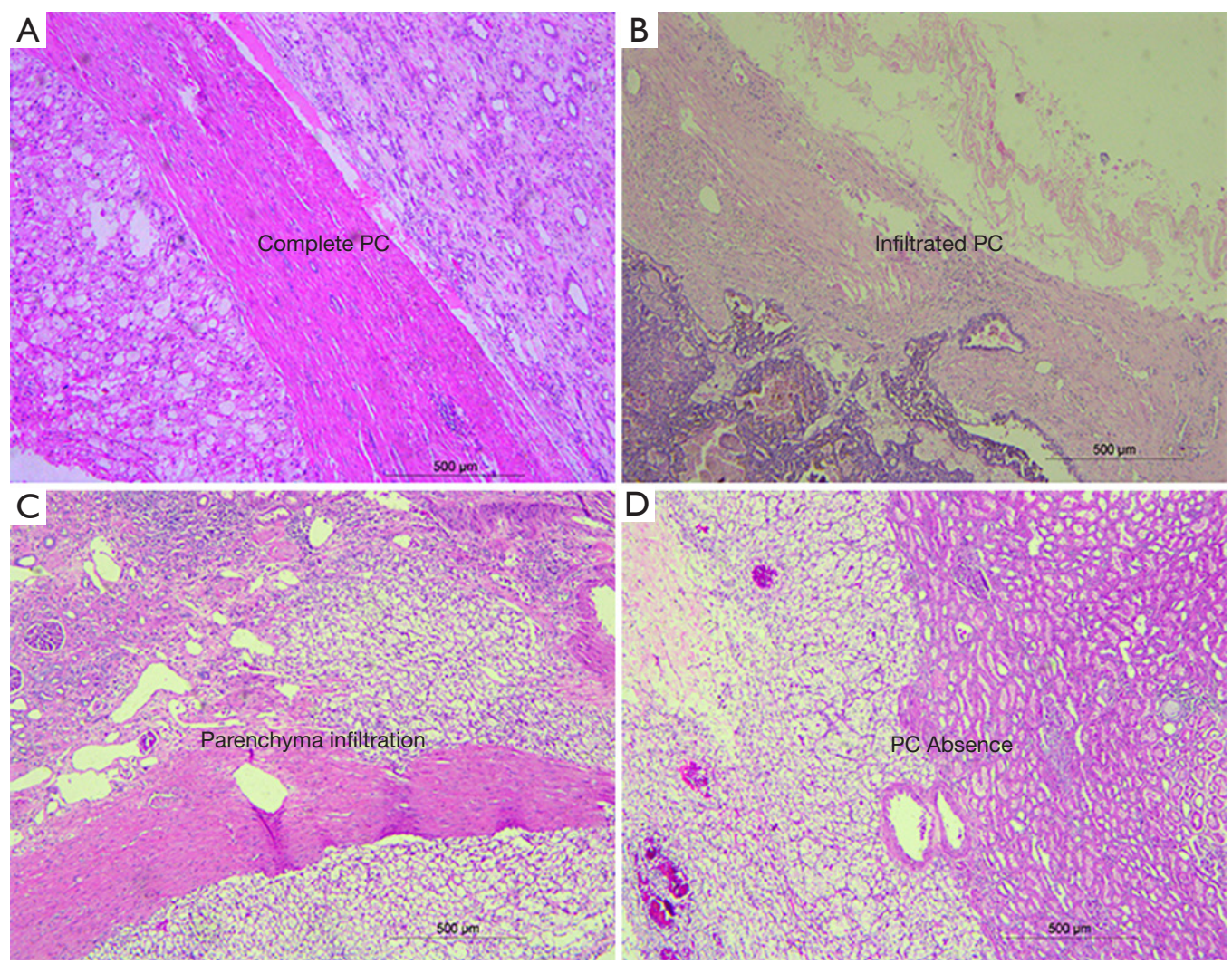

Figure 1 PC status. (A) Complete PC: PC was intact and free from invasion; (B) Infiltrated PC: neoplastic infiltration occurred in the PC but not exceeded it; (C) Parenchyma infiltration: neoplastic infiltration occurred in the peritumoral parenchyma; (D) PC absence: PC was not visible at any point in all slides and the cancer cell directly contacted with the renal parenchyma. PC, pseudocapsule.

package). The discrimination ability of the nomogram was measured by C-index calculated from the area under ROC curves. A calibration curve was plotted to further validate the statistical performance by using Hosmer-Lemeshow test. Decision curve analysis was performed to determine the clinical usefulness by calculating the net benefits at different threshold probabilities. On this basis, we further plotted the clinical impact curve of the nomogram. Finally, we performed survival analysis by using Cox proportional hazard regression analyses. All statistical analyses were performed using SPSS version22 (IBM, Armonk, NY, USA) and $\mathrm{R}$, and all tests were two-sided with a significance level of 0.05 .

\section{Results}

\section{Clinicopatbological characteristics}

Eventually, 170 eligible patients were enrolled with a mean age of 52.56 years. PC invasion occurred in 70 (41.2\%) tumors. All surgical margins were negative which were confirmed by an experienced pathologist. Necrosis on CT scan occurred in 60 (35.3\%) tumors. One hundred thirty-five (79.4\%) tumors were diagnosed as clear cell RCC. Median (IQR) of NLR, PLR, LMR and AGR were 2.41 (1.66-3.46), 119.64 (87.34-160.83), 3.75 (2.80$4.80)$ and $1.60(1.50-1.80)$, respectively. Other detailed clinicopathological data were shown in Table 1.

\section{Associations between systemic inflammatory markers with clinicopathological features}

The optimal cutoffs of NLR, PLR, LMR and AGR were $3.13,113.51,2.41,1.35$, respectively. We found that higher NLR was significantly associated with higher $\mathrm{BMI}(\mathrm{P}=0.007)$ and higher necrosis rates on $\mathrm{CT}$ scan $(\mathrm{P}<0.001)$. Lower AGR was significantly related to older age $(\mathrm{P}=0.005)$. Lower LMR was related to higher necrosis rates on CT scan $(\mathrm{P}=0.042)$. All systemic markers were positively related 
Table 1 Clinicopathological characteristics

\begin{tabular}{|c|c|}
\hline Variables & Number \\
\hline Age, years, mean $\pm S D$ & $52.56 \pm 12.04$ \\
\hline RENAL score, median [range] & $4.1[2-6]$ \\
\hline \multicolumn{2}{|l|}{ Gender, n (\%) } \\
\hline Female & $50(29.4)$ \\
\hline Male & $120(70.6)$ \\
\hline \multicolumn{2}{|l|}{ BMI, n (\%) } \\
\hline$\leq 28 \mathrm{~kg} / \mathrm{m}^{2}$ & $109(64.1)$ \\
\hline$>28 \mathrm{~kg} / \mathrm{m}^{2}$ & $61(35.9)$ \\
\hline \multicolumn{2}{|l|}{ Smoking history, n (\%) } \\
\hline No & $97(57.1)$ \\
\hline Yes & 73 (42.9) \\
\hline \multicolumn{2}{|l|}{ Chronic renal disease, n (\%) } \\
\hline No & $165(97.1)$ \\
\hline Yes & $5(2.9)$ \\
\hline \multicolumn{2}{|l|}{ Hypertension, n (\%) } \\
\hline No & $153(90.0)$ \\
\hline Yes & $17(10.0)$ \\
\hline \multicolumn{2}{|l|}{ Coronary heart disease, n (\%) } \\
\hline No & $160(94.1)$ \\
\hline Yes & $10(5.9)$ \\
\hline \multicolumn{2}{|l|}{ Imaging findings on CT scan } \\
\hline \multicolumn{2}{|l|}{ Tumor size, n (\%) } \\
\hline$\leq 4 \mathrm{~cm}$ & $122(71.8)$ \\
\hline$>4 \mathrm{~cm}$ & $48(28.2)$ \\
\hline \multicolumn{2}{|l|}{ Necrosis, n (\%) } \\
\hline Absent & $110(64.7)$ \\
\hline Yes & $60(35.3)$ \\
\hline \multicolumn{2}{|l|}{ Tumor shape, n (\%) } \\
\hline Regular & $122(71.8)$ \\
\hline Irregular & $48(28.2)$ \\
\hline \multicolumn{2}{|l|}{ Enhancement pattern, n (\%) } \\
\hline Homogeneous & $86(50.6)$ \\
\hline Heterogeneous & $84(49.4)$ \\
\hline
\end{tabular}

Table 1 (continued)
Table 1 (continued)

\begin{tabular}{lc}
\hline Variables & Number \\
\hline Inflammatory markers, median (IQR) & $2.41(1.66-3.46)$ \\
NLR & $3.75(2.80-4.80)$ \\
LMR & $119.64(87.34-160.83)$ \\
PLR & $1.60(1.50-1.80)$ \\
AGR & \\
Histological subtype, $\mathrm{n}(\%)$ & $135(79.4)$ \\
ccRCC & $10(5.9)$ \\
chrRCC & $8(4.7)$ \\
ONC & $17(10.0)$ \\
papRCC & \\
Fuhrman grade, $\mathrm{n}(\%)$ & $131(77.1)$ \\
G1-2 & $39(22.9)$ \\
G3-4 & \\
PC invasion, $\mathrm{n}(\%)$ & $100(58.8)$ \\
Absent & $70(41.2)$ \\
Present & \\
\hline
\end{tabular}

ccRCC, clear cell renal cell carcinoma; chrRCC, chromophobe RCC; papRCC, papillary RCC; ONC, oncocytoma; PC, pseudocapsule; BMI, body mass index; NLR, neutrophillymphocyte ratio; PLR, platelet-lymphocyte ratio; LMR, lymphocyte-monocyte ratio; AGR, albumin-globulin ratio; CT, computed Tomography; SD, standard deviation.

to PC invasion. However, none of them was related to tumor grade, histological subtype, gender, tumor shape or enhancement pattern. These data were shown in Table S2.

\section{Risk factors of PC invasion}

Univariable analyses revealed that larger $\mathrm{BMI}(\mathrm{P}<0.001)$, higher tumor grade $(\mathrm{P}<0.001)$, larger tumor size $(\mathrm{P}=0.002)$, the presence of tumor necrosis on CT scan $(\mathrm{P}<0.001)$, higher NLR $(\mathrm{P}<0.001)$, higher PLR $(\mathrm{P}=0.002)$, lower LMR $(\mathrm{P}<0.001)$ and lower AGR $(\mathrm{P}<0.001)$ were related to $\mathrm{PC}$ invasion (Table 2). Then we performed further multivariable logistic analyses to adjust all potential covariant. We demonstrated that larger BMI [odds ratio (OR) 4.81; $95 \%$ confidence interval $(\mathrm{CI}): 2.01-11.53 ; \mathrm{P}<0.001]$, higher 
Table 2 Logistic regression analyses for predictors of PC invasion

Variables $\quad \frac{\text { Univariable analyses }}{\text { OR }(95 \% \mathrm{Cl}) \mathrm{P} \text { value }} \frac{\text { Multivariable analyses }}{\mathrm{OR}(95 \% \mathrm{Cl}) \mathrm{P} \text { value }}$

Age (years)

$\begin{array}{ccc}<60 & \text { Reference } & \\ \geq 60 & 1.47 & 0.263 \\ & (0.75-2.88) & \end{array}$

Gender

$\begin{array}{lcc}\text { Female } & \text { Reference } & \\ \text { Male } & 0.67 & 0.243 \\ & (0.35-1.31) & \end{array}$

BMI

\begin{tabular}{lcccc}
$\leq 28 \mathrm{~kg} / \mathrm{m}^{2}$ & Reference & \multicolumn{3}{c}{ Reference } \\
$>28 \mathrm{~kg} / \mathrm{m}^{2}$ & 4.46 & $<0.001$ & 4.81 & $<0.001$ \\
& $(2.28-8.70)$ & & $(2.01-11.53)$ &
\end{tabular}

Smoking history

$\begin{array}{ccc}\text { No } & \text { Reference } & \\ \text { Yes } & 0.90 & 0.739 \\ & (0.49-1.67) & \end{array}$

Histological subtype

$\begin{array}{lcc}\text { ccRCC } & \text { Reference } & \\ \text { chrRCC } & 0.95 & 0.926 \\ & (0.34-2.66) & \\ \text { ONC } & 2.14 & 0.348 \\ & (0.44-10.53) & \\ \text { papRCC } & 0.86 & 0.861 \\ & (0.15-4.82) & \end{array}$

Fuhrman grade

\begin{tabular}{ccccc} 
G1-2 & Reference & \multicolumn{3}{c}{ Reference } \\
G3-4 & 4.61 & $<0.001$ & 8.23 & $<0.001$ \\
& $(2.13-9.96)$ & & $(2.83-23.94)$ &
\end{tabular}

Imaging findings

Tumor size

\begin{tabular}{ccccc}
$\leq 4 \mathrm{~cm}$ & Reference & \multicolumn{3}{c}{ Reference } \\
& & & & \\
& 3.01 & 0.002 & 3.13 & 0.021 \\
& $(1.51-6.01)$ & & $(1.19-8.25)$ &
\end{tabular}

Necrosis

\begin{tabular}{lcccc} 
Absent & Reference & \multicolumn{3}{c}{ Reference } \\
& 4.21 & $<0.001$ & 2.55 & 0.043 \\
Present & $(2.16-8.20)$ & & $(1.03-6.30)$ & \\
& & & &
\end{tabular}

Table 2 (continued)
Table 2 (continued)

\begin{tabular}{|c|c|c|c|c|}
\hline \multirow{2}{*}{ Variables } & \multicolumn{2}{|c|}{ Univariable analyses } & \multicolumn{2}{|c|}{ Multivariable analyses } \\
\hline & OR $(95 \% \mathrm{Cl})$ & $P$ value & OR $(95 \% \mathrm{Cl})$ & $P$ value \\
\hline \multicolumn{5}{|l|}{ Tumor shape } \\
\hline Regular & Reference & & & \\
\hline Irregular & $\begin{array}{c}1.03 \\
(0.52-2.23)\end{array}$ & 0.935 & & \\
\hline \multicolumn{5}{|c|}{ Enhancement pattern } \\
\hline Homogeneous & Reference & & & \\
\hline Heterogeneous & $\begin{array}{c}1.26 \\
(0.68-2.33)\end{array}$ & 0.452 & & \\
\hline
\end{tabular}

Inflammatory markers

NLR

$$
\begin{aligned}
& <3.13 \\
& \geq 3.13
\end{aligned}
$$

Reference

$10.79<0.001$

(4.92-23.66)

Reference

$$
\begin{array}{ll}
6.17 \quad 0.002
\end{array}
$$

PLR

$$
\begin{aligned}
& <113.51 \\
& \geq 113.51
\end{aligned}
$$

$\begin{array}{ll}\text { Reference } & \\ 2.78 & 0.002 \\ (1.46-5.27) & \end{array}$

(1.96-19.46)

0.002

\section{LMR}

$$
\begin{aligned}
& \geq 2.41 \\
& <2.41
\end{aligned}
$$

Reference

Reference

$$
\text { A }
$$

4.93
$(2.03-11.94)$

$$
\begin{gathered}
1.94 \\
(0.72-5.26)
\end{gathered}
$$

0.190

AGR

$$
\begin{aligned}
& \geq 1.35 \\
& <1.35
\end{aligned}
$$

Reference

Reference

4.10
$(1.95-8.58)$

$<0.001$

3.21 0.027

ccRCC, clear cell renal cell carcinoma; chrRCC, chromophobe RCC; papRCC, papillary RCC; ONC, oncocytoma; PC, pseudocapsule; NLR, neutrophil-lymphocyte ratio; PLR, plateletlymphocyte ratio; LMR, lymphocyte-monocyte ratio; AGR, albumin-globulin ratio; CT, computed Tomography; BMI, body mass index.

tumor grade (OR 8.23; 95\% CI: 2.83-23.94; $\mathrm{P}<0.001$ ), larger tumor size (OR 3.13; 95\% CI: 1.19-8.25; $\mathrm{P}=0.021$ ), the presence of tumor necrosis (OR 2.55; 95\% CI: $1.03-$ 6.30; $\mathrm{P}=0.043$ ), higher NLR (OR 6.17; 95\% CI: 1.96-19.46; $\mathrm{P}=0.002$ ), and lower AGR (OR 3.21; 95\% CI: 1.14-9.05; $\mathrm{P}=0.027)$ were independent risk factors of $\mathrm{PC}$ invasion. 

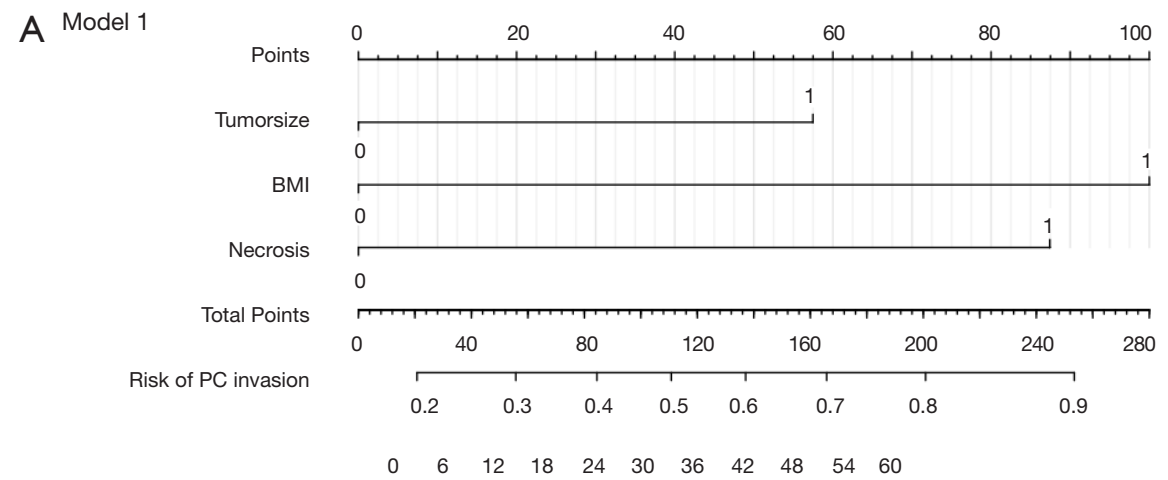

B Model 2
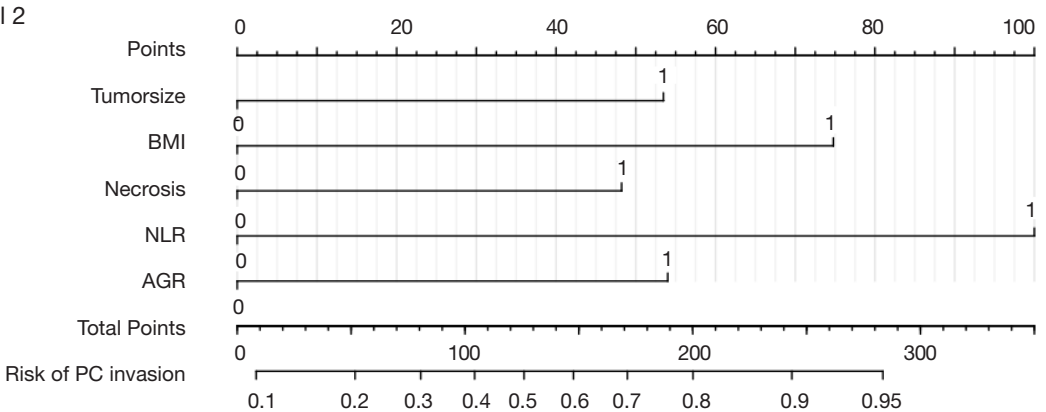

C Model 3
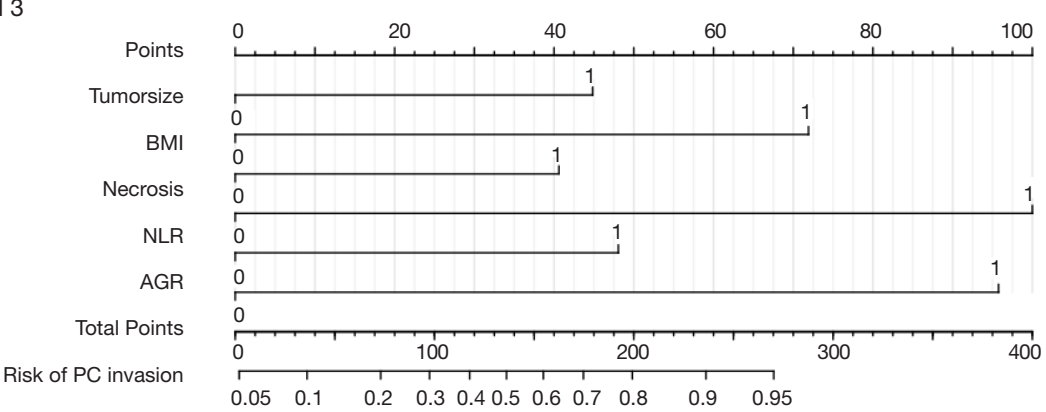

Figure 2 Nomograms predicting PC invasion in patients with localized RCC. (A) Model 1: clinical model incorporating BMI, tumor size and tumor necrosis on CT scans; (B) Model $1+$ inflammatory markers (NLR and AGR); (C) Model 2 + pathological tumor grade. PC, pseudocapsule; RCC, renal cell carcinoma; NLR, neutrophil-lymphocyte ratio; AGR, albumin-globulin ratio; BMI, body mass index.

\section{Development of an individualized nomogram to predict PC status}

Three prediction models were constructed based on these independent risk factors. Model 1 incorporated tumor size, BMI and necrosis on CT scan (Figure 2A). On this basis, we added two systemic inflammatory markers (NLR and AGR) to construct the Model 2 (Figure 2B). In addition, we constructed the Model 3 by incorporating tumor grade on the basis of Model 2 (Figure 2C). The results of discrimination curves demonstrated that the C-index of Model 2 was higher than that of Model 1 (0.85 vs. 0.78)
(Figure 3A). The decision curves further indicated that Model 2 was superior to Model 1 in predicting PC invasion (Figure $3 B$ ). Although the ability of discrimination and clinical decision of Model 3 were slightly superior to Model 2, tumor grade was detected postoperatively. Therefore, we selected Model 2 as the final prediction model.

\section{Statistical performance and clinical use of Model 2}

The Model 2 included NLR, AGR, tumor size, BMI and tumor necrosis on CT scan. We implemented ROC curves 

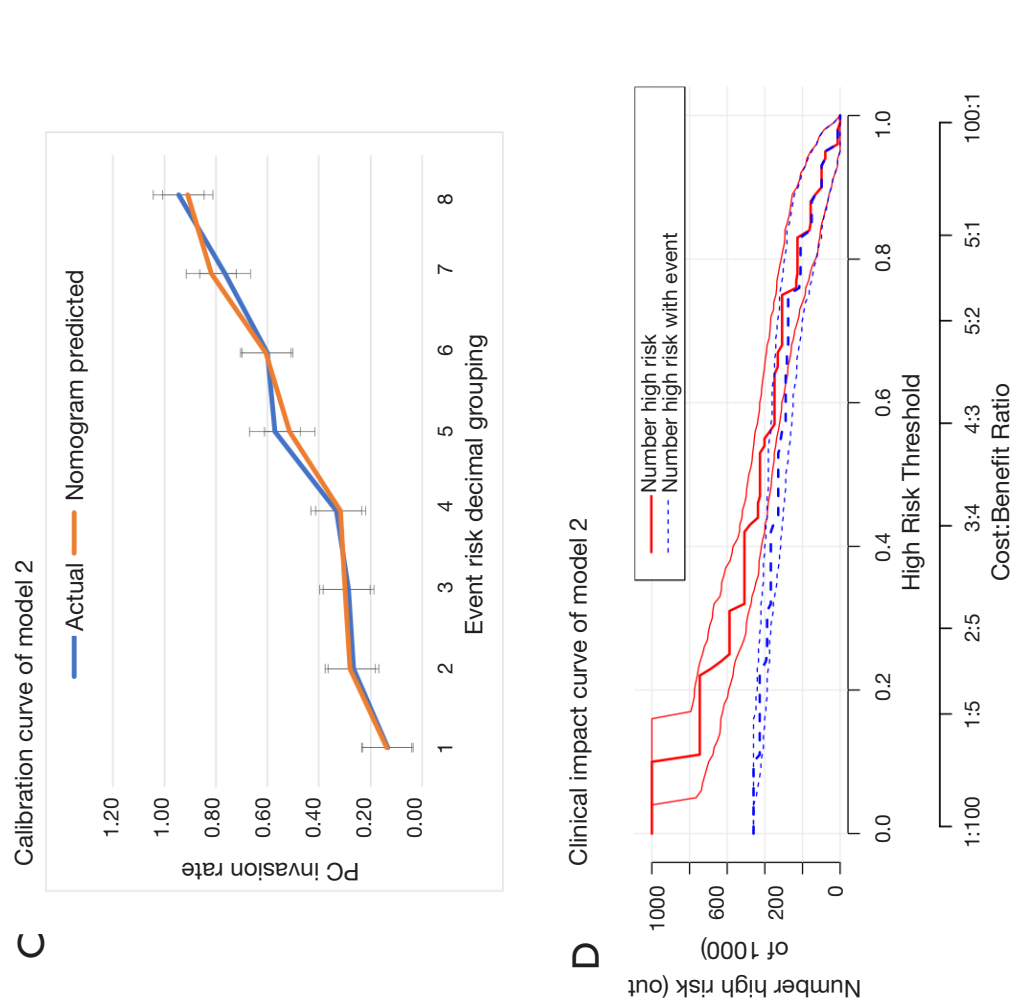

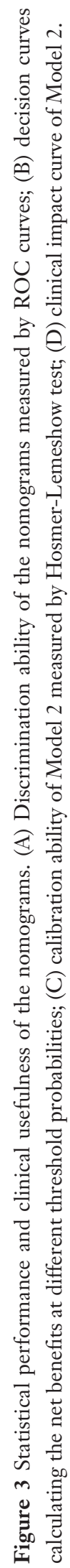
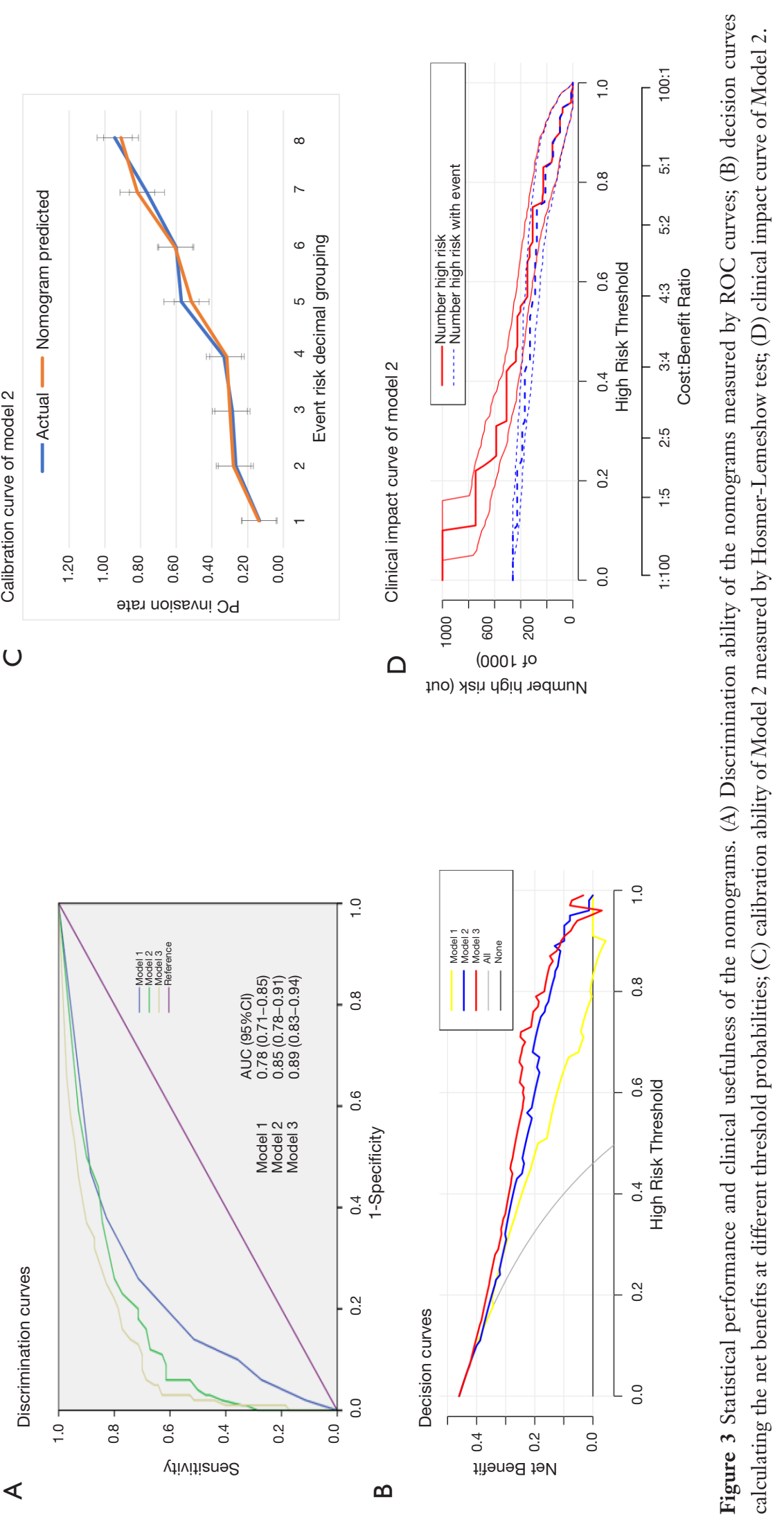
Table 3 Cox regression analyses for prognostic factors of overall survival

\begin{tabular}{|c|c|c|c|c|}
\hline Variables & \multicolumn{2}{|c|}{ Univariable analysis } & \multicolumn{2}{|c|}{ Multivariable analysis } \\
\hline Age ( $\geq 60$ vs. $<60$ years) & $2.51(1.26-4.98)$ & 0.009 & $5.56(2.32-13.13)$ & $<0.001$ \\
\hline Gender (male vs. female) & $1.94(0.80-4.70)$ & 0.142 & & \\
\hline BMI (>28 vs. $\leq 28$ kg/m²) & $4.82(2.29-10.14)$ & $<0.001$ & $16.25(6.14-43.01)$ & $<0.001$ \\
\hline Chronic renal disease (yes vs. no) & $0.87(0.11-6.93)$ & 0.867 & & \\
\hline Hypertension (yes vs. no) & $2.29(0.94-5.64)$ & 0.069 & & \\
\hline Coronary heart disease (yes vs. no) & $0.96(0.21-4.43)$ & 0.960 & & \\
\hline Tumor size (>4 vs. $\leq 4 \mathrm{~cm}$ ) & $4.18(2.09-8.35)$ & $<0.001$ & $12.75(4.76-34.12)$ & $<0.001$ \\
\hline $\begin{array}{l}\text { Enhancement pattern (heterogeneous vs. } \\
\text { homogeneous) }\end{array}$ & $0.55(0.27-1.11)$ & 0.094 & & \\
\hline $\operatorname{NLR}(\geq 3.13$ vs. $<3.13)$ & $7.86(3.65-16.94)$ & $<0.001$ & $28.25(7.44-107.25)$ & $<0.001$ \\
\hline PLR ( $\geq 113.51$ vs. <113.51) & $1.17(0.58-2.32)$ & 0.665 & & \\
\hline $\operatorname{LMR}(<2.41$ vs. $\geq 2.41)$ & $2.80(1.36-5.78)$ & 0.005 & $0.82(0.32-2.13)$ & 0.696 \\
\hline AGR (<1.35 vs. $\geq 1.35)$ & $2.95(1.49-5.87)$ & 0.002 & $2.24(0.88-5.70)$ & 0.091 \\
\hline PC invasion (present vs. absent) & $2.45(1.23-4.96)$ & 0.010 & $31.49(7.94-124.84)$ & $<0.001$ \\
\hline Fuhrman grade (G3-4 vs. G1-2) & $3.51(1.76-6.97)$ & $<0.001$ & $18.90(6.78-52.64)$ & $<0.001$ \\
\hline
\end{tabular}

ccRCC, clear cell renal cell carcinoma; NLR, neutrophil-lymphocyte ratio; PLR, plate-lymphocyte ratio; LMR, lymphocyte-monocyte ratio; AGR, albumin-globulin ratio; BMI, body mass index.

to measure the predictive value of this final multivariable model. The C-index of this model was 0.85 (95\% CI, 0.78$0.91)$. The sensitivity was $62 \%$ and specificity was $94 \%$. The calibration curve for the risk of PC invasion demonstrated good agreement between prediction and observation (Figure $3 C$ ). The Hosmer-Lemeshow test yielded a nonsignificant statistic $(\mathrm{P}=0.602)$, which indicated that Model 2 was well fitting. The decision curve demonstrated that if the threshold probability of a patient or doctor is $10 \%$, using Model 2 to predict PC invasion added more benefit than either the treat-all-patients or the treat-none scheme. Finally, its' clinical value was further validated with the clinical impact curve (Figure 3D).

\section{Prognostic factors of overall survival}

Median follow-up time was 70 (IQR, 63-80) months.
Thirty-three patients died during the follow-up period. The 3 - and 5 -year overall survival rates were $90 \%$ and $81 \%$ respectively. Table 3 showed the results of survival analyses. After we adjusted all covariates in the multivariable cox regression analyses, we found that older age [hazard ratio (HR) 5.56; 95\% CI: 2.32-13.13; $\mathrm{P}<0.001$ ], larger BMI (HR 16.25; 95\% CI: 6.14-43.01; $\mathrm{P}<0.001$ ), larger tumor size (HR 12.75; 95\% CI: 4.76-34.12; $\mathrm{P}<0.001)$, the presence of tumor necrosis (HR 2.90; 95\% CI: 1.34-6.28; $\mathrm{P}=0.007$ ), higher NLR (HR 28.25; 95\% CI: 7.44-107.25; $\mathrm{P}<0.001$ ), higher tumor grade (HR 18.90; 95\% CI: 6.78-52.64; $\mathrm{P}<0.001$ ) and PC invasion (HR 31.49; $95 \%$ CI: 7.94-124.84; $\mathrm{P}<0.001$ ) were independent adverse prognostic factors of overall survival.

\section{Discussion}

Whether it is necessary to excise a layer of renal parenchyma 
around the cancer during NSS remains controversial. Some evidences support that preserving all non-neoplastic renal parenchyma via TE procedure achieves comparable oncological control and long-term survival with better renal function recovery when it compares with standard NSS $(2,3,17,18)$. However, some researchers suggest that TE surgery may cause poorer prognosis $(7,8)$. They think that excising a layer of healthy parenchyma around the cancer via standard NSS procedure is crucial for better survival by achieving negative surgical margin. Given these controversies over the oncological control of TE procedure, urologists should be carefully selecting ideal patients for TE surgery.

Cho et al. performed a prospective multicenter study (10). They found that $58.4 \%$ renal tumors were completely surrounded by a continuous PC. The larger tumor size $(>4 \mathrm{~cm})$ and non-clear cell subtype were risk factors for $\mathrm{PC}$ invasion. Therefore, they hold the view that the TE surgery should be performed with extreme care. Some other studies also explored the risk factors of PC invasion. Overall, there were several potential risk factors such as tumor stage, grade, tumor size, necrosis, and histological subtype $(10,19,20)$. However, most of these predictors were confirmed postoperatively. We should identify some preoperative predictors for PC invasion, which can help urologists chose the appropriate surgical approach. Therefore, we generated a systemic inflammatory markerbased nomogram for the preoperative prediction of PC invasion in patients with localized RCC. This nomogram incorporated three parts of the inflammatory markers (NLR and AGR), BMI and CT imaging features (tumor size and tumor necrosis). All items successfully stratified tumors according to their risk of $\mathrm{PC}$ invasion. A high C-index of 0.85 and a well-fitting calibration curve indicated that this nomogram was robust. To further justify its clinical usefulness, we assessed whether nomogramassisted decisions would improve patient outcomes by performing decision curve analysis. The decision curves suggested that if the threshold probability was $10 \%$, using this inflammatory nomogram to predict PC invasion added more benefit than either the treat-all-patients or the treatnone scheme. Above all, the current easy-to-use nomogram facilitated urologists to identify the best candidates to receive TE surgery.

The accuracy of magnetic resonance imaging (MRI) to identify PC invasion in renal tumors is higher than that of CT (21). However, in clinical practice, CT is the first and most accurate method for diagnosing renal cancer. In addition, the cost of MRI is more expensive than CT and the examination time is longer. Overall, CT is of higher value than MRI for early diagnosis and clinical decision making, which makes most doctors and patients prefer CT examination. Therefore, all patients in our study received CT examination. We demonstrated that several imaging features on CT scans, such as tumor size and necrosis, were able to help urologists preliminarily predict PC status. Similarly, Wei et al. found that CT could differentiate the tumor grade, PC status, and tumor necrosis of renal cancer. However, neither CT nor MRI can detect the latent micro PC invasion. To increase the predictive accuracy and identify micro PC invasion, we combined these imaging features with pre-treatment systemic inflammatory markers.

Boissier et al. summarized that NLR was a strong prognostic factor of renal cancer and NLR could improve the statistical performance of predictive nomograms used in renal cancer (22). Viers et al. demonstrated that NLR could facilitate urologists to distinguish benign and malignant renal masses (23). An elevated NLR predicted advanced RCC pathology, such as higher-grade and more aggressive histologic subtypes. Similarly, Chen et al. found that preoperative lower AGR was associated with poorer prognosis and more advanced pathology, such as tumor necrosis and tumor size (24). In addition, they demonstrated that AGR could improve the accuracy of prognostic nomogram for over survival. So far, no previous study explores the role of NLR and AGR in predicting PC status. Here, we found that NLR and AGR were independent risk factors of PC invasion. The addition of NLR and AGR to the nomogram along with other risk factors (BMI, imaging features) significantly improved the statistical performance of the nomogram and increased its clinical net benefit.

We recognized several limitations of our study. Firstly, this study was retrospective, single-central and nonrandomized; Secondly, all included tumors were localized RCC, which may result in selection bias. Thirdly, some preoperative hematological parameters were collected from other medical centers, which may cause measurement bias. The hematological parameters might be affected by some latent sickness. Fourthly, we did not analyze the biological mechanism under the association between these systemic inflammatory markers and PC invasion.

\section{Conclusions}

We constructed a nomogram that incorporated both the systematic inflammatory markers and clinical risk factors. 
It can be conveniently used to preoperatively predict the individualized risk of PC invasion and identify the best candidates to receive TE surgery.

\section{Acknowledgments}

We sincerely thank You-e He for editing the language of this article.

Funding: This work was supported by the National Key R\&D Program of China (2016YFC0902603), the National Natural Science Foundation of China (grant numbers 81572523, 81700665), the Hunan Province Funds for Distinguished Young Scientists of China (grant number 2016JJ1026).

\section{Footnote}

Conflicts of Interest: All authors have completed the ICMJE uniform disclosure form (available at http://dx.doi. org/10.21037/tau.2020.01.26). XZ serves as an unpaid editorial board member of Translational Andrology and Urology from Mar 2019 to Feb 2021. The other authors have no conflicts of interest to declare.

Ethical Statement: The authors are accountable for all aspects of the work in ensuring that questions related to the accuracy or integrity of any part of the work are appropriately investigated and resolved. This study obtained approval from our institutional ethical review board (Ethical approval ID: 201912530). The informed consent was waived for this study.

Open Access Statement: This is an Open Access article distributed in accordance with the Creative Commons Attribution-NonCommercial-NoDerivs 4.0 International License (CC BY-NC-ND 4.0), which permits the noncommercial replication and distribution of the article with the strict proviso that no changes or edits are made and the original work is properly cited (including links to both the formal publication through the relevant DOI and the license). See: https://creativecommons.org/licenses/by-ncnd/4.0/.

\section{References}

1. Ljungberg B, Albiges L, Abu-Ghanem Y, et al. European Association of Urology Guidelines on Renal Cell Carcinoma: The 2019 Update. Eur Urol 2019;75:799-810.
2. Simmons MN, Hillyer SP, Lee BH, et al. Functional recovery after partial nephrectomy: effects of volume loss and ischemic injury. J Urol 2012;187:1667-73.

3. Mir MC, Campbell RA, Sharma N, et al. Parenchymal volume preservation and ischemia during partial nephrectomy: functional and volumetric analysis. Urology 2013;82:263-8.

4. Carini M, Minervini A, Masieri L, et al. Simple enucleation for the treatment of PT1a renal cell carcinoma: our 20year experience. Eur Urol 2006;50:1263-8; discussion 1269-71.

5. Carini M, Minervini A, Lapini A, et al. Simple enucleation for the treatment of renal cell carcinoma between 4 and $7 \mathrm{~cm}$ in greatest dimension: progression and long-term survival. J Urol 2006;175:2022-6; discussion 2026.

6. Dell'Atti L, Scarcella S, Manno S, et al. Approach for Renal Tumors With Low Nephrometry Score Through Unclamped Sutureless Laparoscopic Enucleation Technique: Functional and Oncologic Outcomes. Clin Genitourin Cancer 2018;16:e1251-6.

7. Kwon EO, Carver BS, Snyder ME, et al. Impact of positive surgical margins in patients undergoing partial nephrectomy for renal cortical tumours. BJU Int 2007;99:286-9.

8. Bradshaw A, Eldefrawy A, Uzzo R, et al. Negative impact of positive margins in partial nephrectomy in stage 1 renal cell carcinoma: A multicenter analysis. Eur Urol 2019;18:e424-5.

9. O'Donnell PW, Manivel JC, Cheng EY, et al. Chemotherapy influences the pseudocapsule composition in soft tissue sarcomas. Clin Orthop Relat Res 2014;472:849-55.

10. Cho S, Lee JH, Jeon SH, et al. A prospective, multicenter analysis of pseudocapsule characteristics: Do all stages of renal cell carcinoma have complete pseudocapsules? Urol Oncol 2017;35:370-8.

11. Cao J, Zhu X, Zhao X, et al. Neutrophil-to-Lymphocyte Ratio Predicts PSA Response and Prognosis in Prostate Cancer: A Systematic Review and Meta-Analysis. PLoS One 2016;11:e0158770.

12. Jiang $\mathrm{Y}, \mathrm{Xu} \mathrm{H}$, Jiang $\mathrm{H}$, et al. Pretreatment neutrophillymphocyte count ratio may associate with gastric cancer presence. Cancer Biomark 2016;16:523-8.

13. Wu S, Zhao X, Wang Y, et al. Pretreatment NeutrophilLymphocyte Ratio as a Predictor in Bladder Cancer and Metastatic or Unresectable Urothelial Carcinoma Patients: a Pooled Analysis of Comparative Studies. Cell Physiol Biochem 2018;46:1352-64. 
14. Song X, Zhu H, Pei Q, et al. Significance of inflammationbased indices in the prognosis of patients with nonmetastatic colorectal cancer. Oncotarget 2017;8:45178-89.

15. Wu S, Wan Q, Xu R, et al. Systematic review and metaanalysis of the prognostic value of preoperative plateletto-lymphocyte ratio in patients with urothelial carcinoma. Oncotarget 2017;8:91694-702.

16. Wei J, Zhao J, Zhang X, et al. Analysis of dual energy spectral CT and pathological grading of clear cell renal cell carcinoma (ccRCC). PLoS One 2018;13:e0195699.

17. Minervini A, Ficarra V, Rocco F, et al. Simple enucleation is equivalent to traditional partial nephrectomy for renal cell carcinoma: results of a nonrandomized, retrospective, comparative study. J Urol 2011;185:1604-10.

18. Mukkamala A, Allam CL, Ellison JS, et al. Tumor enucleation vs sharp excision in minimally invasive partial nephrectomy: technical benefit without impact on functional or oncologic outcomes. Urology 2014;83:1294-9.

19. Minervini A, Campi R, Di Maida F, et al. Tumorparenchyma interface and long-term oncologic outcomes after robotic tumor enucleation for sporadic renal cell carcinoma. Urol Oncol 2018;36:527.e1-11.

20. Minervini A, di Cristofano C, Lapini A, et al. Histopathologic analysis of peritumoral pseudocapsule and surgical margin status after tumor enucleation for renal cell carcinoma. Eur Urol 2009;55:1410-8.

21. van Oostenbrugge TJ, Runneboom W, Bekers E, et al. MRI as a tool to assess surgical margins and pseudocapsule features directly following partial nephrectomy for small renal masses. Eur Radiol 2019;29:509-16.

22. Boissier R, Campagna J, Branger N, et al. The prognostic value of the neutrophil-lymphocyte ratio in renal oncology: A review. Urol Oncol 2017;35:135-41.

23. Viers BR, Thompson RH, Lohse CM, et al. Pretreatment neutrophil-to-lymphocyte ratio predicts tumor pathology in newly diagnosed renal tumors. World J Urol 2016;34:1693-9.

24. Chen Z, Shao Y, Yao H, et al. Preoperative albumin to globulin ratio predicts survival in clear cell renal cell carcinoma patients. Oncotarget 2017;8:48291-302.
Cite this article as: $\mathrm{Hu}$ J, Chen J, Li H, He T, Deng H, Gong G, Cui Y, Liu P, Ren W, Zhou X, Li C, Zu X. A preoperative nomogram predicting the pseudocapsule status in localized renal cell carcinoma. Transl Androl Urol 2020;9(2):462-472. doi: 10.21037/tau.2020.01.26 
Patients with localized RCC treated with NSS $\mathrm{n}=250$

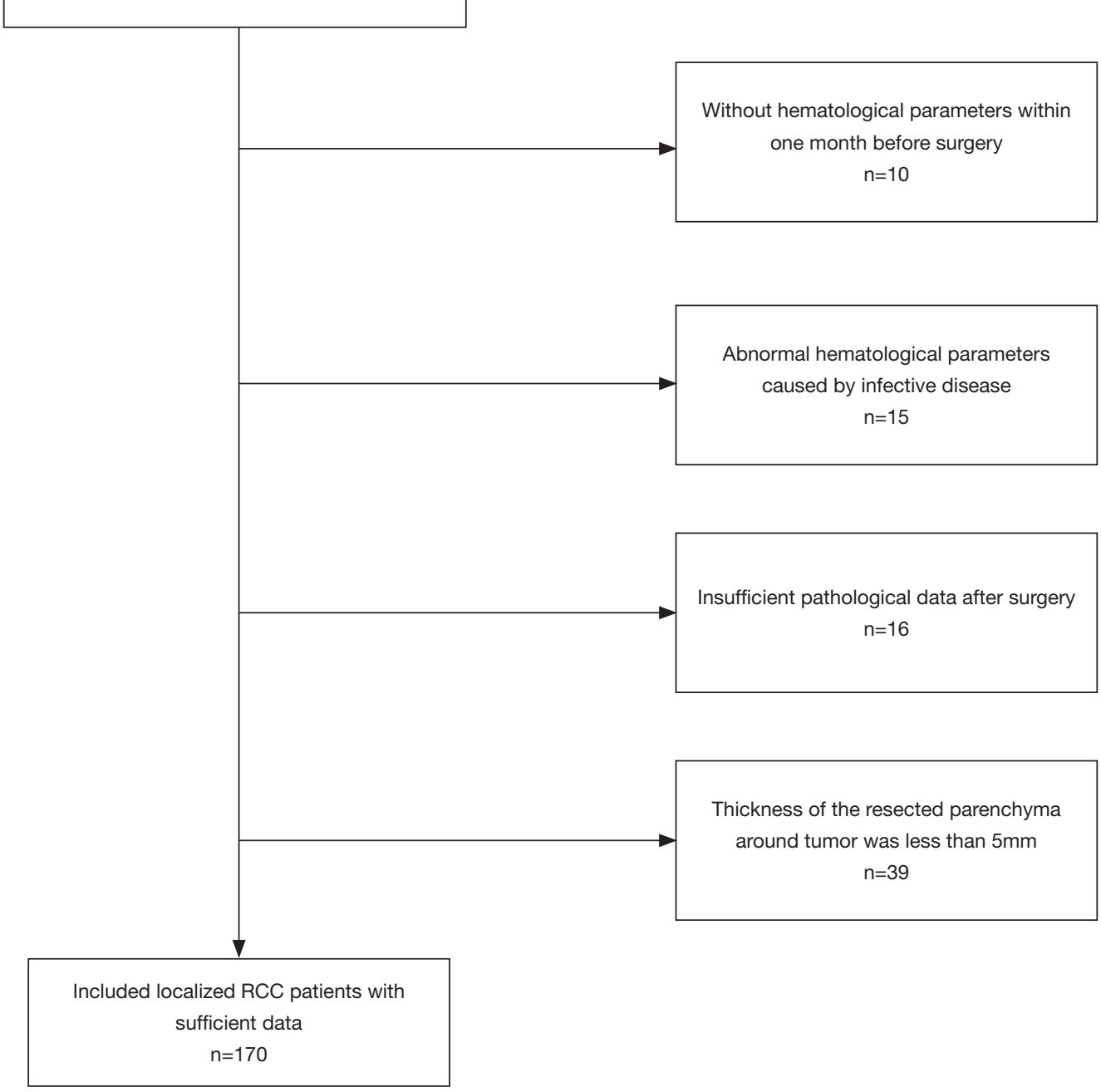

Figure S1 Flow diagram of patient inclusion. RCC, renal cell carcinoma. 

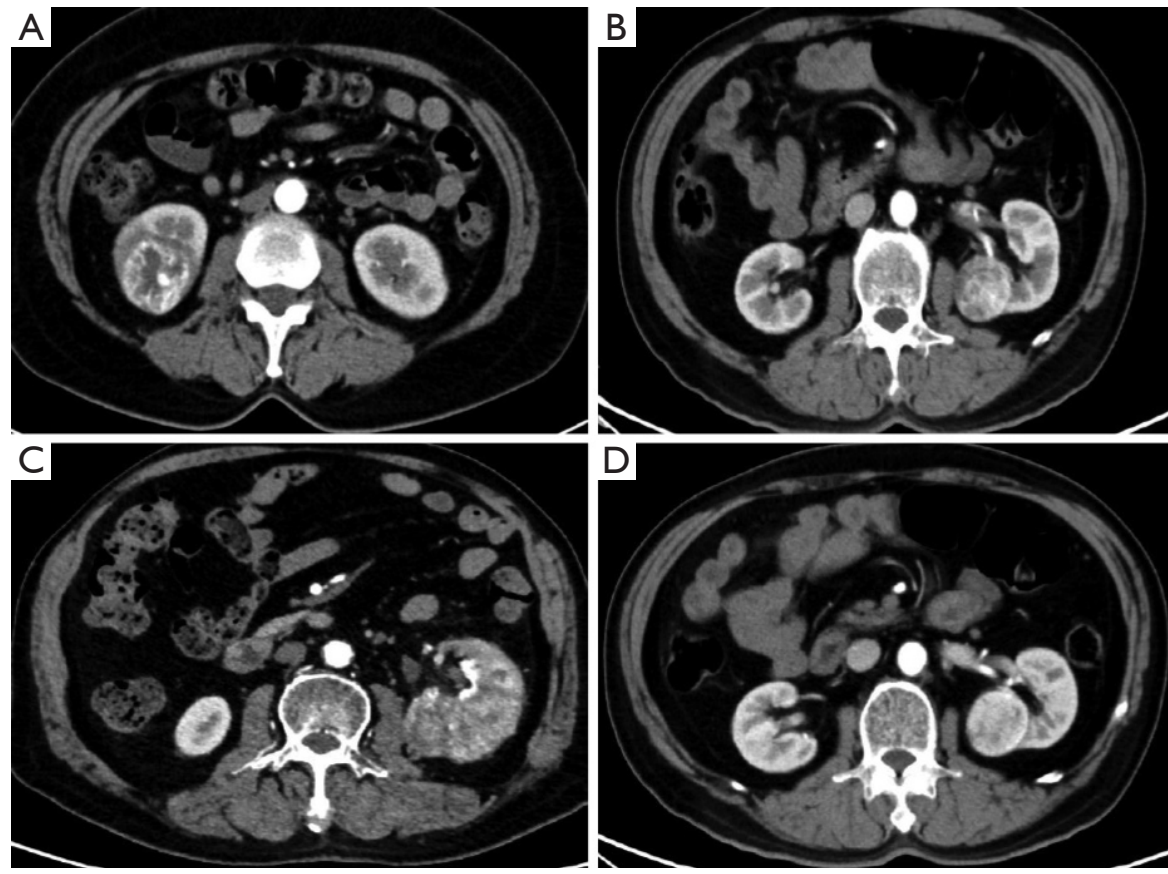

Figure S2 Imaging features on CT scan. (A) Necrosis; (B) heterogeneous enhancement pattern; (C) irregular tumor shape; (D) regular tumor shape.

Table S1 The normal references of hematological parameters

\begin{tabular}{lc}
\hline Hematological parameters & Normal reference \\
\hline Neutrophile granulocyte $\left(\times 10^{9} / \mathrm{L}\right)$ & $1.8-6.3$ \\
Lymphocyte $\left(\times 10^{9} / \mathrm{L}\right)$ & $1.1-3.2$ \\
Monocyte $\left(\times 10^{9} / \mathrm{L}\right)$ & $0.1-0.6$ \\
Blood platelet $\left(\times 10^{9} / \mathrm{L}\right)$ & $125-135$ \\
Albumin $(\mathrm{g} / \mathrm{L})$ & $40-55$ \\
Globulin $(\mathrm{g} / \mathrm{L})$ & $20-40$ \\
\hline
\end{tabular}




\begin{tabular}{|c|c|c|c|c|c|c|c|c|c|c|c|c|}
\hline \multirow{2}{*}{ Variables } & \multicolumn{3}{|c|}{ NLR } & \multicolumn{3}{|c|}{ PLR } & \multicolumn{3}{|c|}{ AGR } & \multicolumn{3}{|c|}{ LMR } \\
\hline & $<3.13$ & $\geq 3.13$ & $P$ value & $<113.51$ & $\geq 113.51$ & $P$ value & $\geq 1.35$ & $<1.35$ & $P$ value & $\geq 2.41$ & $<2.41$ & $P$ value \\
\hline Age (years) & & & 0.334 & & & 0.499 & & & 0.005 & & & 0.084 \\
\hline$<60$ & 88 & 34 & & 54 & 68 & & 99 & 23 & & 105 & 17 & \\
\hline$\geq 60$ & 31 & 17 & & 24 & 24 & & 29 & 19 & & 36 & 12 & \\
\hline Gender & & & 0.142 & & & 0.301 & & & 0.801 & & & 0.258 \\
\hline Female & 39 & 11 & & 26 & 24 & & 37 & 13 & & 44 & 6 & \\
\hline Male & 80 & 40 & & 52 & 68 & & 91 & 29 & & 97 & 23 & \\
\hline BMI & & & 0.007 & & & 0.109 & & & 0.145 & & & 0.127 \\
\hline$\leq 28$ & 84 & 25 & & 55 & 54 & & 86 & 23 & & 94 & 15 & \\
\hline$>28$ & 35 & 26 & & 23 & 38 & & 42 & 19 & & 47 & 14 & \\
\hline Smoking history & & & 0.478 & & & 0.642 & & & 0.729 & & & 0.144 \\
\hline No & 70 & 27 & & 46 & 51 & & 74 & 23 & & 84 & 13 & \\
\hline Yes & 49 & 24 & & 32 & 41 & & 54 & 19 & & 57 & 16 & \\
\hline \multicolumn{13}{|l|}{ Imaging findings } \\
\hline Tumor size & & & 0.087 & & & 0.089 & & & 0.956 & & & 0.713 \\
\hline$\leq 4 \mathrm{~cm}$ & 90 & 32 & & 51 & 71 & & 92 & 30 & & 102 & 20 & \\
\hline$>4 \mathrm{~cm}$ & 29 & 19 & & 27 & 21 & & 36 & 12 & & 39 & 9 & \\
\hline Necrosis & & & $<0.001$ & & & 0.622 & & & 0.120 & & & 0.042 \\
\hline Absent & 89 & 21 & & 52 & 58 & & 87 & 23 & & 96 & 14 & \\
\hline Present & 30 & 30 & & 26 & 34 & & 41 & 19 & & 45 & 15 & \\
\hline Tumor shape & & & 0.372 & & & 0.726 & & & 0.215 & & & 0.149 \\
\hline Regular & 83 & 39 & & 57 & 65 & & 95 & 27 & & 98 & 24 & \\
\hline Irregular & 36 & 12 & & 21 & 27 & & 33 & 15 & & 43 & 5 & \\
\hline Enhancement pattern & & & 0.547 & & & 0.434 & & & 0.657 & & & 0.588 \\
\hline Homogeneous & 62 & 24 & & 42 & 44 & & 66 & 20 & & 70 & 16 & \\
\hline Heterogeneous & 57 & 27 & & 36 & 48 & & 62 & 22 & & 71 & 13 & \\
\hline Histological subtype & & & 0.469 & & & 0.137 & & & 0.816 & & & 0.597 \\
\hline $\mathrm{ccRCC}$ & 96 & 39 & & 67 & 68 & & 103 & 32 & & 110 & 25 & \\
\hline chrRCC & 5 & 5 & & 3 & 7 & & 7 & 3 & & 8 & 2 & \\
\hline ONC & 5 & 3 & & 1 & 7 & & 5 & 3 & & 7 & 1 & \\
\hline papRCC & 13 & 4 & & 7 & 10 & & 13 & 4 & & 16 & 1 & \\
\hline Fuhrman grade & & & 0.360 & & & 0.743 & & & 0.155 & & & 0.514 \\
\hline G1-2 & 94 & 37 & & 61 & 70 & & 102 & 29 & & 110 & 21 & \\
\hline G3-4 & 25 & 14 & & 17 & 22 & & 26 & 13 & & 31 & 8 & \\
\hline PC invasion & & & $<0.001$ & & & 0.002 & & & $<0.001$ & & & $<0.001$ \\
\hline Absent & 89 & 11 & & 56 & 44 & & 86 & 14 & & 92 & 8 & \\
\hline Present & 30 & 40 & & 22 & 48 & & 42 & 28 & & 49 & 21 & \\
\hline
\end{tabular}

ccRCC, clear cell renal cell carcinoma; chrRCC, chromophobe RCC; papRCC, papillary RCC; ONC, oncocytoma; PC, pseudocapsule; NLR, neutrophil-lymphocyte ratio; PLR, platelet-lymphocyte ratio; LMR, lymphocyte-monocyte ratio; AGR, albumin-globulin ratio; CT, computed Tomography; SD, standard deviation; BMI, body mass index. 